\title{
Clique-based Location Estimations for Wireless Sensors in GPS-free Environments
}

\author{
Patryk Mazurkiewicz and Kin K. Leung \\ Electrical \& Electronic Engineering Department, Imperial College, London, U.K. \\ \{patryk.mazurkiewicz06, kin.leung\}@imperial.ac.uk
}

\begin{abstract}
In this paper we present a distributed, self-organizing, localization method for wireless sensors and a scheme for reducing position estimate errors by employing a novel concept of cliques of nodes.

We describe an applicable theoretical foundation of peer-topeer distance-vector (DV) exchange algorithm, which can operate without any manual pre-configuration. This method allows for propagating the complete information of a reference system (i.e., axes orientation and origin position) using angle and range measurements.

In order to take advantage of possible spatial-diversity of measurements and to produce enhanced position estimates, we propose here an enhancement method which is based on groups of interconnected nodes (cliques) to combine localization measurements using a minimum-square-error (MSE) criterion and spatial transformations. This enables propagation of the reference system among all the nodes in a clique more accurately than by regular peer-to-peer distance-vector exchanges. Computer simulation reveals that the new cliquebased error mitigating scheme reduces the localization error by $65 \%$ for a typical underground tunnel setting.
\end{abstract}

Keywords: localization, positioning, location estimation, wireless sensor.

\section{INTRODUCTION}

Localization of nodes in wireless sensor networks (WSNs) has attracted considerable research attention recently. Accurate location estimations of sensors are required in numerous industrial, military and public applications of WSN. Sensor networks used for monitoring purposes in general require accurate positioning information in order to link the sensing data to the place where it is gathered. From a wider perspective, localization in WSN can serve as a basis for solving a number of networking problems, like scheduling of the transmission [1] and geographical routing [2].

WSNs used for monitoring applications have to be designed to meet the specific requirements and characteristics of the application environments. This fact is strongly reflected in the indoor systems where operating constraints can vary significantly from one application scenario to another. The particularly harsh environments, like tunnels,

This work is funded by U.K. EPSRC Research Grant EP/D076838/1 entitled: "Smart Infrastructure: Wireless Sensor Network System for Condition Assessment and Monitoring of Infrastructure." impose constraints on the possible network topology. For such long structures, the WSN represents a linear topology [3]. This limits the usability of certain localization methods or reduces dramatically their accuracy. In fact, the public literature has few studies about methods dedicated to localization in tunnels and thus the challenges for accurate localization techniques remain to be addressed.

The major issue with localization in linear networks is that the geographical information needs to be transmitted over multiple hops, which can distort this information extensively [4]. When passing the location information through the network, the inaccuracy of position estimates accumulates and gives increasingly erroneous results at every following hop. The second potential issue specific for linear topologies is that nodes tend to have a relatively small number of neighboring nodes comparing to other networks with nodes uniformly distributed in space. This limits the use of nonDV-exchange methods, e.g. [5], [6], [7]. Those methods in linear networks, even if possible to employ at all, would suffer as the number of neighboring nodes is smaller than 8 , an optimal number for satisfactory performance [8]. To adequately address these outstanding design issues, we propose an algorithm implementing the DV-exchange localization method and extend it into a clique-based localization technique to improve accuracy. This work thus specifically addresses the inherent issues of relatively sparse network topologies.

In this paper we focus on a localization method that uses angle and range measurements in order to establish a networkwide coordinate system. The main contribution of the paper is elaborating a clique-based algorithm that refines the measurements using the redundancy in groups of interconnected nodes. This greatly improves the accuracy of localization comparing to algorithms not using cliques. The other contribution is elaborating the peer-to-peer localization algorithm that is self-organizing. This algorithm uses the angle and range measurements in order to pass a 3-D reference system in the peer-to-peer mode, between two nodes, without any precalibration and without using earth-gravity direction as in [4].

The remaining part of the paper is organized as follows. Related work is presented in section II. The algorithm for peer-to-peer reference system exchange is described in section III. Section IV explains the clique-based algorithm for propagating the coordinate system in an accurate way, 
which is one-to-many mode. In $\mathrm{V}$ we show how to apply the calculated corrections to the measurements in order to present them in the global reference system. Simulation results showing the performance of the proposed algorithm and discussion of the results are presented in section VI.

\section{RELATED WORK}

In this section, we give the background for the techniques that can be used as angular and ranging measurement methods in the DV-exchange localization method. We also specify limitations of the localization methods of significant importance to this work which were not mentioned in the introduction.

\section{A. Spatial measurements}

DV-exchange method is based on the measurements of distance and angle between sensors (nodes). The methods for obtaining these quantities in the peer-to-peer mode can be Time of Arrival (ToA) and Angle of Arrival (AoA). In this work we assume using antenna arrays for obtaining these quantities, but we base on the assumption that we are given the values of angles and distance with some error without analyzing the performance of a particular measurement technique.

Commonly used ToA technique requires extremely accurate time-measurements due to high speed of radio wave. Since this is a problematic issue, methods for 'common viewpoint measurements' have been developed [5] for improving the measurement accuracy. Also, tight timing synchronization among nodes such as synchronization based on the out-band radio signalling [9] mitigates the error generated in measurements. There is also room for improvement in the sensor timing by e.g., implementing on-chip atomic clock [10].

Time Difference of Arrival (TDoA) based on differences in time of arrival of a radio-wave from different nodes is not considered here because of its requirement of having at least 4 reference nodes in order to locate a sensor in the 3-D environments. The other variation of TDoA measuring the difference between arrival times of radio and acoustic signals is interference-prone and is unsuitable in noisy environments. Nevertheless, cliques of nodes, which are elaborated in section IV, may be capable of using TDoA within them, but this is not considered in this paper.

The Received Signal Strength (RSS) method is not assumed to be a good candidate to be taken into account here, since it depends on the a priori knowledge of propagation model, which may be unknown and variant in tunnels.

Angle of arrival measurement techniques have been well developed for antenna arrays [11]. There is a constant advance in the algorithm design of AoA techniques. Support Vector Machines have been proposed recently as a candidate for solving beamforming and AoA problems in antenna arrays [12]. This technique requires a priori training phase, but its advantage is the accuracy despite a small set of training data. Nevertheless any training phase is inconvenient in our application. A technique called modified beamspace Music algorithm [13] does not require the training phase and is designed for narrow-band applications, which is consistent with typical sensor usage in WSNs. It is then a matter of construction issues to build sensors capable of measuring 3-D AoA based on the technique from [13].

\section{B. Localization techniques}

Positioning method efficiently overcoming the problem of an unknown propagation model in tunnels was proposed in [14]. It uses a neural network to learn the response fingerprint of a sensor being in a certain location in the tunnel. Nevertheless, similar methods cannot be used in the selforganized, training-less system like the one under consideration in this work. This is so because the method in [14] aims at localizing mobile sensors in the known environment. In contrast, the operating environment considered in this work is not known in advance.

The DV-exchange is also used in the method proposed in [4]. However, the technique in [4] requires that one of the coordinates of the reference system is aligned with the direction of earth gravity. The new method in this work does not have that requirement. Furthermore, the nodes' coordinate systems are mirrored in the technique in [4]. The new technique introduced here avoids that and thus reducing computational overhead. Last but not least, our new algorithm makes use of the clique concept to "smooth" out estimation errors by MSE. To the best of our knowledge, such a technique is a new idea for node localization in WSNs.

\section{BASIC DV-EXCHANGE ALGORITHM}

This section shows the algorithm for propagating the coordinate system in a peer-to-peer mode without employing additional nodes in the exchange. One of the nodes knows its position and the other obtains the coordinate system from the former.

We regard two particular reference systems aligned if their corresponding coordinate axes point at the same directions. By corrections we mean the angular differences by which a coordinate system needs to be rotated in order to align them to another system. An unknown node is a node with its position unknown.

\section{A. Aligning the axes}

A peer-to-peer DV exchange explained in this section occurs between nodes $a$ (knowing the full information about the frame of reference) and $b$ (being an unknown node). Node $b$ uses $a$ prim reference system which is unaligned with this of $a$. 


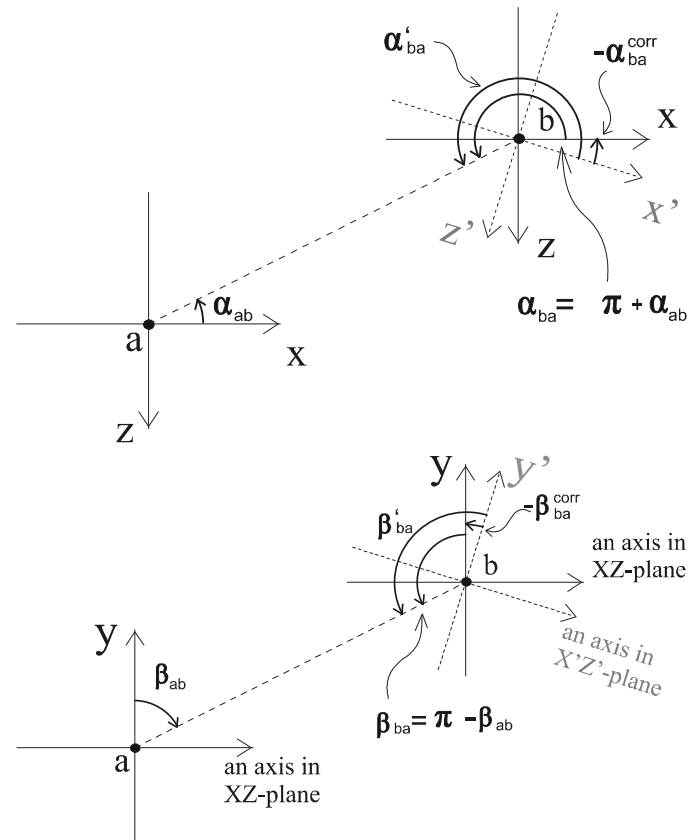

Figure 1. Corrections of azimuth and elevation of ' $b$ ' with reference to ' $a$ '.

Angles measured from $a$ to $b$ have the indexes ${ }_{a b}$ and viceversa. Azimuth angle is denoted as $\alpha$, while elevation is $\beta$.

Finding corrections for the reference system of a node $b$ from spatial measurements between the nodes $a$ and $b$ is shown in Figure 1.

$$
\begin{aligned}
& \alpha_{b a}-\alpha_{a b}=\pi \quad \text { and } \quad \beta_{a b}+\beta_{b a}=\pi \\
& \alpha_{b a}=\alpha_{b a}^{\prime}+\alpha_{b a}^{c o r r} \quad \text { and } \quad \beta_{b a}=\beta_{b a}^{\prime}+\beta_{b a}^{c o r r} \\
& \alpha_{b a}^{c o r r}=\pi+\alpha_{a b}-\alpha_{b a}^{\prime} \quad \text { and } \beta_{b a}^{c o r r}=\pi-\beta_{a b}-\beta_{b a}^{\prime}
\end{aligned}
$$

If the reference systems for both nodes are aligned, the azimuth and elevation angles satisfy (1). Otherwise, the corrections of the prim-reference system are defined in (2) and the measured angles satisfy (3).

\section{B. Finding the position}

The distance vector between the nodes $a$ and $b$ in the global reference system is defined unequivocally as $\vec{d}=\left(x_{a b}, y_{a b}, z_{a b}\right)$ where

$$
\begin{aligned}
& x_{a b}=d \cos \alpha_{a b} \sin \beta_{a b}, \\
& y_{a b}=d \cos \beta_{a b}, \\
& z_{a b}=-d \sin \alpha_{a b} \sin \beta_{a b} .
\end{aligned}
$$

Adding $\vec{d}$ to the position vector of node $a$ gives the position vector of node $b$ :

$$
\left(x_{a}, y_{a}, z_{a}\right)+\left(x_{a b}, y_{a b}, z_{a b}\right)=\left(x_{b}, y_{b}, z_{b}\right) .
$$

The application of these corrections to transform the node's physical measurements is discussed in section $\mathrm{V}$.

\section{Clique-Based Error Mitigation For DV-Exchange}

This section describes an algorithm to compute enhanced corrections by combining multiple "redundant" measurements obtained by a group of interconnected nodes. This leads to more accurate location estimates of all nodes in the group. Such a group is called a clique for the reason that it comprises solely of sensors mutually connected with each other. In other words, sensor nodes of a clique are one hop away from each other.

\section{A. Number of clique members}

Consider that sensor nodes are deployed on the ground, ceiling and/or wall of a tunnel which has a designated

gateway node responsible for collecting data from the sensors for transfer onto the backhaul network. The gateway is also a reference node, which is position- and orientation-aware. For reliability reasons, each sensor should have at least two disjoint routes to reach the gateway node. For "linear" networks meeting the redundancy requirement, geometrical analysis of their topology shows that the networks can be typically divided into overlapping cliques of three or more nodes.

\section{B. Combining redundant measurements}

This sub-section describes a method for combining redundant measurements obtained within a clique.

A particular difficulty when merging the redundant distance-vector measurements from different nodes is that they are all obtained in different frames of reference, so they need to be transformed in order to be comparable.

Since all $n$ nodes in a clique are mutually connected, they can measure the locations of each other. In other words, all nodes can perform measurements leading to constructing a physical map of the clique. There will be one map per node since all nodes are ToA- and AoA-capable. Since measurements involve errors, all maps represent erroneous physical locations of nodes in the clique. Each of the $n$ nodes is represented $n$ times by $n-1$ measured position-vectors and by its own local position. The key question is how to combine the $n$ three-dimensional maps of the whole clique into an accurate map.

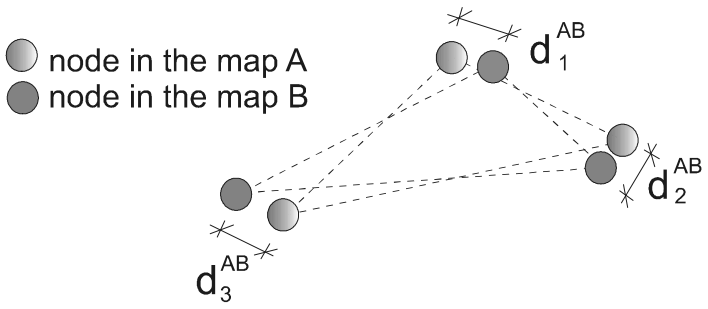

Figure 2. Two maps of three nodes aligned to each other by rotating and shifting with reference to each other. The sum $\sum d_{k}$ is minimized. 


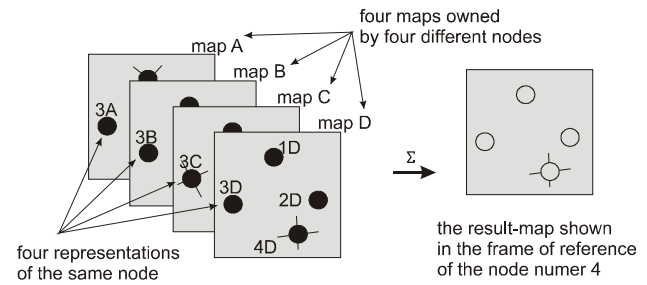

Figure 3. Several maps are put on top of each other, rotated and shifted to obtain the best fit for all of them and subsequently to produce a precise global geographical map.

The proposed algorithm to combine the $n$ maps of a clique consists of rotating and transferring the maps in order to achieve the greatest concentration of representations of nodes, as exemplified in Figure 3. This process resembles aligning a graph of a simple geometric shape with a transparent sheet having roughly the same drawing on it. In this example, these two

sheets (graphs) are put together: one on another, and the top sheet is transferred or rotated in order to align the graphs. Similar action is performed by the algorithm on the maps of a clique's topology, but in three dimensions. Also, there are $n$ 'sheets' (maps) to align.

We propose to use the minimum-square-error (MSE) criterion to find the best fitting of different erroneous maps of a clique. MSE is proposed since the problem to be solved is finding a unique set of points that are represented by numerous erroneous measurements per each point. The measurements are scattered in some focus-areas due to the measurement error. In general, MSE minimizes the sum of distances (i.e. errors) between the multiple instances of measurements of each single point (each node) thus producing a map that is a compromise between all the maps.

All nodes perform the measurements in their local frames of reference. The result of this step is that every $k$-th sensor being a member of a clique has a set $X^{k}$ of distance vectors $X_{j}^{k}$ of all other nodes and a position vector of themselves for $j=1$ to $n$. Sets $X^{k}$ of coordinates are called 'maps' here.

The relationship between the points representing the same sensor node on different maps is:

$$
X_{i}^{k}=T X_{i}^{l}+D
$$

where $X_{i}^{k}$ and $X_{i}^{l}$ are position vectors of $i$-th point in $k$-th or $l$ th reference system, $T$ is a rotation matrix rotating $l$-th 'map' by the angle representing the difference between the coordinate systems of $k$-th and $l$-th sensors, and $D$ is a vector representing a transfer of the origin of $l$-th frame of reference to the origin of $k$-th reference system. Elementary rotation matrices are given by [15] and we need to choose any two of three because that is enough to cover any orientation. In order to solve our problem we want to represent all points in the same reference system, hence the indexes of (6) are: $k<l$ and $i, k, l \in\{1 . . n\}$.
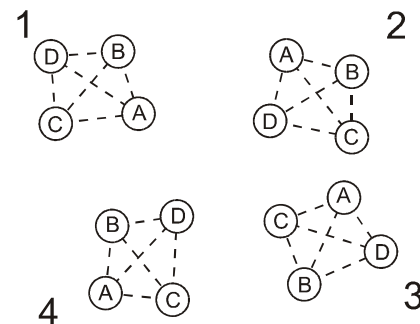

Figure 4. Schematic result of putting maps 'onto' each other. 1-4 are node indices. A-D are representations belonging to maps A-D. Bars represent distances being summed up in (7).

After applying (6) to all maps to produce representations of all position vectors $X_{i}^{l}$ in the same arbitrary chosen frame of reference, we can build an objective function to perform MSE. The variables to sum up and minimize are distances between all representations corresponding to one node. The objective function to minimize is therefore given by:

$$
\sum d^{2}=\sum\left(X_{i}^{k}-X_{i}^{l}\right)^{2}=f\left(\left(\begin{array}{c}
\alpha_{1 m} \\
\beta_{1 m} \\
x_{1 m} \\
y_{1 m} \\
z_{1 m}
\end{array}\right)\right)
$$

where $\alpha_{1 m}, \beta_{1 m}, x_{1 m}, y_{1 m}, z_{1 m}$ are corrections of $m$-th frame of reference, $\mathrm{m} \neq 1$, and they are parameters of the minimized function. Indexes are: $i \in\{1 . . n\}, k<l, k \in\{1 . . n-1\}, l \in\{2 . . n\}$, $m \in\{2 . . n\}$. The position vectors $X_{i}^{k}$ and $X_{i}^{l}$ are either nodes' absolute position in their own local reference system (if $k=i$ or $l=i$ ) or functions of $\alpha_{1 m}, \beta_{1 m}, x_{1 m}, y_{1 m}$ and $z_{1 m}$ (otherwise). Note that the objective function is $5(n-1)$ dimensional because the variables of this function are differences between: (a) any arbitrary chosen local reference system and (b) all $n-1$ rest of the reference systems. The initial values for eventual numerical algorithm solving this problem are the values obtained by the algorithm discussed in Section III. A centralized method (within the area of a clique) for solving such a problem is the steepest gradient method [16].

\section{CORRECting The MEASUREMENTS}

A sensor needs to add the corrections to its measurements in order to use the propagated global reference system, which is as follows.

Sensor node $b$ obtains its corrections for its reference system, namely: $\alpha_{b a}^{\text {corr }}, \beta_{b a}^{\text {corr }},(x, y, z)^{\mathrm{T}}$, and updates its measurements by transforming them in the following way. Any distance vector $\vec{d}$ measured in the local reference system is transformed into vector $\vec{D}$ in the corrected reference system by a transformation in (8): 


$$
\begin{aligned}
& \vec{D}=\vec{d} R_{y}\left(-\alpha_{b a}^{\text {corr }}\right) R_{z}\left(\beta_{b a}^{\text {corr }} \cos \alpha_{b a}^{\text {corr }}\right)+(x, y, z)^{T} \\
& R_{y}(\lambda)=\left[\begin{array}{ccc}
\cos (\lambda) & 0 & -\sin (\lambda) \\
0 & 1 & 0 \\
\sin (\lambda) & 0 & \cos (\lambda)
\end{array}\right], \quad R_{z}=\left[\begin{array}{ccc}
\cos (\lambda) & \sin (\lambda) & 0 \\
-\sin (\lambda) & \cos (\lambda) & 0 \\
0 & 0 & 1
\end{array}\right]
\end{aligned}
$$

where $\mathrm{R}_{\mathrm{y}}(\gamma)$ and $\mathrm{R}_{\mathrm{z}}(\gamma)$ of $(9)$ are rotation matrices.

\section{Simulation Results}

We use a $\mathrm{C}++$ simulation to examine the performance of the proposed scheme. Our simulation model considers a 500 meters long tunnel where nodes are installed in different configurations. Average distance between two senor nodes is 10 meters. We assume the distance measurement error of $30 \%$ of the actual distance and angular error of $\pm 7.5^{\circ}$. Both errors are uniformly distributed as in [17].

We examine the efficiency of our method as a function of the clique size, which is a crucial factor for the system performance. If a clique size is equal to 2 , then the proposed clique-based algorithm is equivalent to the peer-to-peer DVexchange algorithm.

The greater is the size of cliques, the more improvement of the error mitigation capability can be achieved. This is so because measurement errors are "averaged out" by the MSE within the cliques. The more measurements are available, the less importance has the erroneous part of the measurements. When measurement errors at various nodes are not correlated, as intuitively expected, the clique-based method performs well. We believe that this may well be the case in many practical situations, although the error correlation strongly depends on the radio propagation and it is a subject of on-going research in [3].

The average and variance of location estimation errors in a tunnel at a distance of 500 meters away from the beacon node (e.g., gateway node) are shown in Figure 5. The flooding algorithm, which is a baseline for comparison done in the figure, bases on the assumption that each node adjusts and corrects its coordinate system relaying solely on information from the neighboring node that has the least number of hops

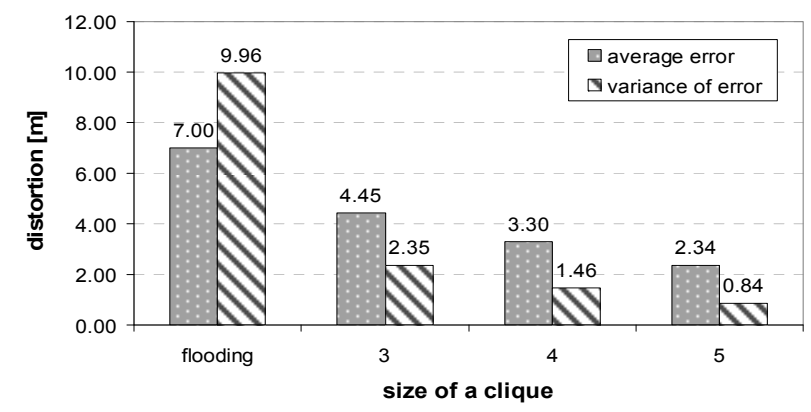

Figure 5. Average and variance of location errors as a function of the clique size at a distance 500 meters away from the beacon (reference) node.

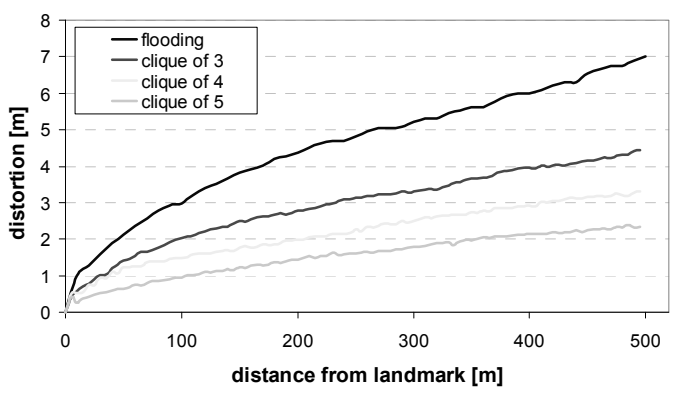

Figure 6. Average location error with reference to the distance from the beacon node for different clique sizes.

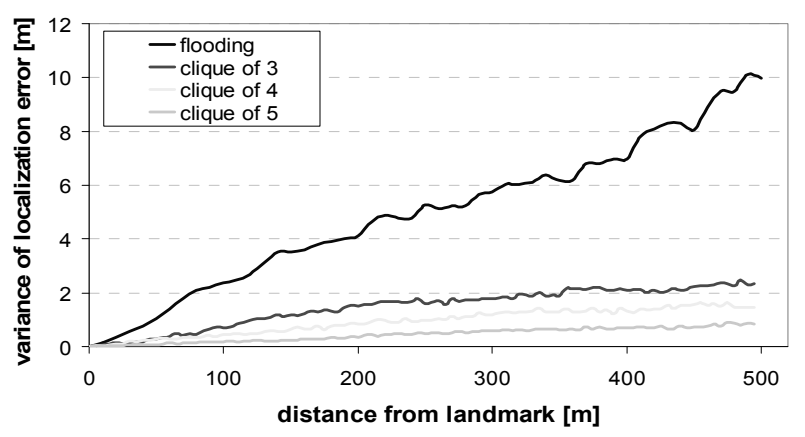

Figure 7. Variance of location error with reference to the distance from the beacon node for different clique sizes.

to the beacon node. As shown in the figure, the proposed algorithm that uses the clique concept can reduce location errors better than the flooding algorithm. Moreover, the error is further reduced as the clique size increases.

With the beacon (reference) node at the beginning of the tunnel, Figure 6 and Figure 7 present the average and variance of the location errors as a function of the node position along the tunnel regarding the clique size. Note that without any technique to control the error propagation in the flooding algorithm, both the average and variance of the location errors increase steadily. To contrast, the proposed method can effectively control the error propagation by combining redundant measurements carried out in cliques.

\section{CONCLUSION AND FUTURE WORK}

We have proposed a new localization algorithm based on distance-vector exchange and a clique-based technique using MSE for accuracy enhancements. The algorithm use peer-to-peer angular and ranging measurements and allow for propagating the

reference system in the infrastructure-less sparse networks of sensors. The algorithm is self-organizing without a need for training or pre-configuration and scalable as computational complexity mainly depends on the number of neighboring nodes, not the total number of nodes in the WSN.

Our simulation results indicate that the new algorithm yields satisfactory location estimates in a typical tunnel 
setting where the estimate error can be improved by as much as $65 \%$ when compared to a standard localization algorithm without considering the error propagation. For the specific 500-meter tunnel setting simulated, the proposed algorithm can estimate node location within an average accuracy of 2.5 meters.

We plan to extend this work in two major directions leading to implementation. Firstly, there is a need for more general description of the algorithm in order to extend the applicability to more complex topologies than linear, and in order to enable it to use many beacon nodes. The more general description would also facilitate finding out the complexity and the overhead of this algorithm. Secondly, we are going to investigate the behavior and characteristics of antenna arrays that could be possibly embedded in sensors in order to gather data for our algorithm. The overall goal of the works is to implement the algorithm and perform the field tests.

\section{ACKNOWLEDGEMENTS}

The authors are grateful to Dr A. Gkelias for his valuable comments to improve presentation of this paper.

\section{REFERENCES}

[1] S.M.Hur, S.Mao, Y.T.Hou, K.Nam, and J.H.Reed, "A LocationAssisted MAC Protocol for Multi-Hop Wireless Networks," in IEEE Wireless Communications and Networking Conference 2007, pp. 322-327.

[2] Z.Sun, R.Yu, and S.Mei, "Robust power-aware routing in wireless sensor networks with special concern about localization error," in AsiaPacific Conference on Communications 2006, pp. 1-5.

[3] N.Graham, K.K.Leung, and et al., "Smart Infrastructure: Wireless sensor network system for condition assessment and monitoring of infrastructure," EPSRC Research Grant EP/D076838/1 http://gow.epsrc.ac.uk/ViewGrant.aspx?GrantRef=EP/D076838/1, accessed on 10 October 2007.

[4] A.Magnani and K.K.Leung, "Self-organized, scalable GPS-free localization of wireless sensors," in IEEE Wireless Communication and Networking Conference Hong Kong, China, 2007.
[5] C.Yan and H.H.Fan, "Asynchronous self-localization of sensor networks with large clock drift," in Fourth Annual International Conference on Mobile and Ubiquitous Systems, Networking \& Services, 6-10 August 2007, pp: 1-8.

[6] L.Zhang, Q.Cheng, Y.Wang, and S.Zeadally, "Landscape: a high performance distributed positioning scheme for outdoor sensor networks," in IEEE International Conference on Wireless And Mobile Computing, Networking And Communications, vol. 3, 2005, pp. 430-437.

[7] D.Niculescu and B.Nath, "Geometry and positioning: Error characteristics of ad hoc positioning systems (APS)," in Proceedings of The ACM International Symposium on Mobile Ad Hoc Networking and Computing, Roppongi Hills, Tokyo, Japan, 2004, pp. 20-30.

[8] V.P.Sadaphal and B.Jain, "Localization accuracy and thershold network density for tracking sensor networks," in IEEE International Conference on Personal Wireless Communications, 2005, pp. 408-412.

[9] R.Mangharam, A.Rowe, and R.Rajkumar, "FireFly Sensor Platform for Multi-hop Networking," in Real-Time Systems Journal accessible at http://www.andrew.cmu.edu/user/rahulm/docs/CMU_FireFly_Overvie w.pdf, 2006, accessed on January 6, 2008.

[10] R.Lutwak, A.Rashed, M.Varghese, G.Tepolt, J.Leblanc, M.Mescher, D.K.Serkland, and G.M.Peake, "The Miniature Atomic Clock - PreProduction Results," in IEEE International Frequency Control Symposium Joint with the 21st European Frequency and Time Forum, 2007, pp. 1327-1333.

[11] L.C.Godara, "Application of antenna arrays to mobile communications. II. Beam-forming and direction-of-arrival considerations," in Proceedings of the IEEE, vol. 85, 1997, pp. 1195-1245.

[12] X.Nan, C.G.Christodoulou, M.Martinez-Ramon, and T.Ozdemir, "Antenna array processing for radar applications using support vector machines," in IEEE Antennas and Propagation Society International Symposium 2006, pp. 1295-1298.

[13] V.I.Vasyiyshyn, "Antenna array signal processing with high-resolution by modified beamspace music algorithm," in 6th International Conference on Antenna Theory and Techniques, 2007, pp. 455-457.

[14] C.Nerguizian, C.Despins, and S.Affes, "Geolocation in mines with an impulse response fingerprinting technique and neural networks," in IEEE Transactions on Wireless Communications, vol. 5, 2006, pp. 603-611.

[15] E.W.Weisstein, "Rotation Matrix," in From MathWorld--A Wolfram Web Resource, 1999, available at http://mathworld.wolfram.com/RotationMatrix.html, accessed on Dec 20, 2007.

[16] C.Bishop, "Neural Networks for Pattern Recognition," Oxford, UK, Clarendon Press, 1997.

[17] X.Ji and H.Zha, "Robust sensor localization algorithm in wireless adhoc sensor networks," in Proceedings of 12th International Conference on Computer Communications and Networks, 2003, pp. 527-532. 